\title{
Hollow Gold Nanoshells Encapsulated in PNIPAM Nanoparticles
}

Sofía Alejandra Tovar-Cabrera ${ }^{1}$, Reynaldo Esquivel ${ }^{1}$, Cindy Alejandra Gutiérrez-Valenzuela ${ }^{2}$, Pedro Hernández ${ }^{1}$, Paul Zavala-Rivera ${ }^{2}$, Iván Eleazar Moreno- Cortez ${ }^{3}$, Roberto Guzman-Zamudio ${ }^{4}$ and Armando Lucero-Acuña ${ }^{2 *}$

1. Nanotechnology Graduate Program, Department of Physics, University of Sonora, MEXICO.

2. Department of Chemical and Metallurgical Engineering, University of Sonora, Hermosillo, MEXICO.

3. Faculty of Mechanical and Electrical Engineering, Autonomous University of Nuevo Leon, Nuevo Leon, MEXICO.

4. Department of Chemical and Environmental Engineering, University of Arizona, Tucson, USA.

In recent decades, nanostructured materials have been of great interest for applications in several areas for technological developments [1]. Research in medical applications have led to the potential use of nanotechnology for the diagnosis, treatment and the monitoring of diseases, improving routes of administration, dosage, and reducing side effects. Nanoparticles of noble metals like gold or silver have become highly relevant due to the multiple possibilities of interactions with biological systems [2]. Also, these metallic nanoparticles present optical properties which depend on the size and shape of them. Metallic nanoparticles can be prepared with different structures, including solid nanoparticles, hollow core nanoshells, and nanorods, among others. Hollow gold shells (HGS) can be prepared by a wellknown method involving a galvanic replacement of silver for gold. According to literature, it is a highly scalable method that utilizes minimal amounts of toxic reagents; also, the HGS core can be adjusted in size and thickness depending on the silver/gold reagent ratios [2,3]. Smart polymers have also been of great interest in medical applications due to their ability of conformational response. An example of an intelligent polymer is poly(isopropylacrylamide) (PNIPAM) which responds to external stimuli such as light and temperature modifying its structure [4]. Nanoparticles of PNIPAM (PNP) prepared by free radical polymerization are reported in literature [5]. In this work, HGS were prepared and encapsulated in PNP (HGS-PNP). HGS were prepared by galvanic replacement on silver nanoparticles (AgNP). Then, HGS were encapsulated in PNP by means of a free radical polymerization of NIPAM.

HGS having surface plasmon resonance (SPR) around $800 \mathrm{~nm}$ were synthesized first by the preparation of AgNP. This particles are synthesized after the overnight reduction of silver nitrate $\left(\mathrm{AgNO}_{3}\right)$ by sodium borohydride $\left(\mathrm{NaBH}_{4}\right)$ in the presence of sodium citrate at $80^{\circ} \mathrm{C}$ [2]. AgNP were used as the initial template to obtain $\mathrm{HGS}$ via the galvanic replacement upon the addition of concentrated $\mathrm{HAuCl}_{4}$ (Fig. 1). The HGS-PNP nanocomposite synthesis was prepared by dissolving a HGS solution in a isopropylacrylamide (NIPAM) and N,N'-methylenebisacrylamide (BIS) under magnetic stirring for 6 hours. Different concentrations of HGS solutions were used: $6.5 \times 10^{-12} \mathrm{M}\left(\mathrm{C}_{1}\right), 5.0 \times 10^{-12} \mathrm{M}\left(\mathrm{C}_{2}\right)$, and $2.6 \times 10^{-12} \mathrm{M}\left(\mathrm{C}_{3}\right)$. Temperature was then raised to $70^{\circ} \mathrm{C}$ and sodium dodecyl sulfate was added. A nitrogen atmosphere was maintained for 15 minutes, a $60 \mathrm{mM}$ ammonium persulfate (APS) solution was added, and the solution was maintained under agitation for 30 minutes. Nanoparticle characterization was performed using a zetasizer Nano ZS equipment (Malvern). When encapsulated in PNP, the resulting HGS-PNP sizes ranged from 171.43 to $267.90 \mathrm{~nm}$, corresponding with the decrease in concentration of HGS added in the formulations. However, PDI values decreased with the HGS concentrations from values from 0.29 to 0.17 as the concentration of HGS decreased. Zeta potentials for HGS is $-27.03 \mathrm{mV}$ while the zeta potential of HGS-PNP is around $-4 \mathrm{mV}$ for all formulations. The SPR peaks obtained for the HGS-PNP showed a peak in the range of the SPR of HGS, but with a 
displacement in the SPR peak (Fig. 2). This displacement could be attributed to the presence of the PNIPAM polymer. The sample of HGS-PNP with $\mathrm{C}_{3}$ does not present the SPR of HGS, mainly because the concentration used in the formulation. The obtained results are consistent with others reported in literature $[2,3,6]$. Surface morphology of the nanoparticles was studied by scanning transmission electron microscopy (STEM) using a JEOL JSM-7800F. Samples were placed in a formvar carbon film and a solution $0.25 \%$ of phosphotungstic acid was added for staining. Figure 3 and 4 show HGS and HGS-PNP micrographs, respectively. Sizes correspond to the results obtained by DLS.

[1] P. Boisseau and B. Loubaton, Comptes Rendus Physics 12 (2011), p. 620.

[2] B. G. Prevo et al., Small 4 (2008), p. 1183.

[3] R. C. Carrillo-Torres et al., RSC Advances, 6 (2016), p. 41304.

[4] R. R. Letfullin, C. B. Iversen, and T. F. George, Nanomedicine: Nanotechnology, Biology and Medicine 7 (2011), p. 137.

[5] R. Esquivel et al., e-Polymers (2017) in press.

[6] T. Pham et al., Langmuir, 18 (2002), p. 4915.

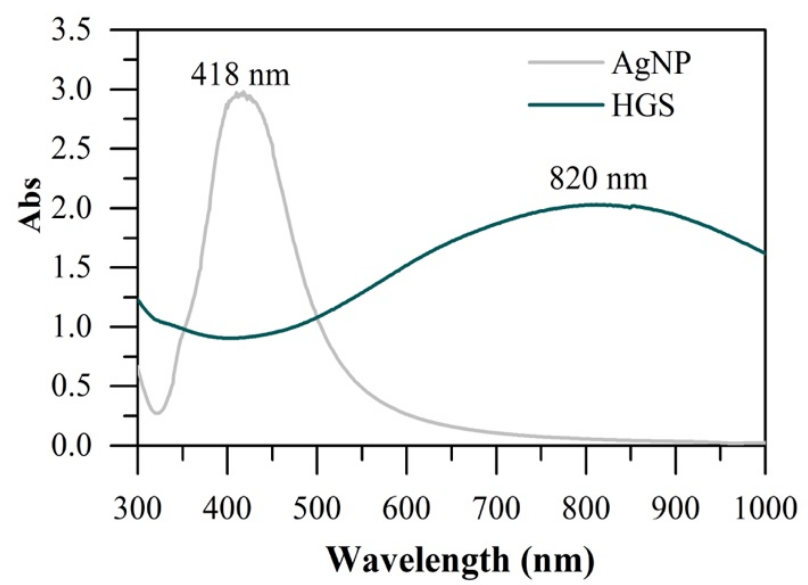

Figure 1. UV-VIS Absorption spectra for AgNP and HGS.

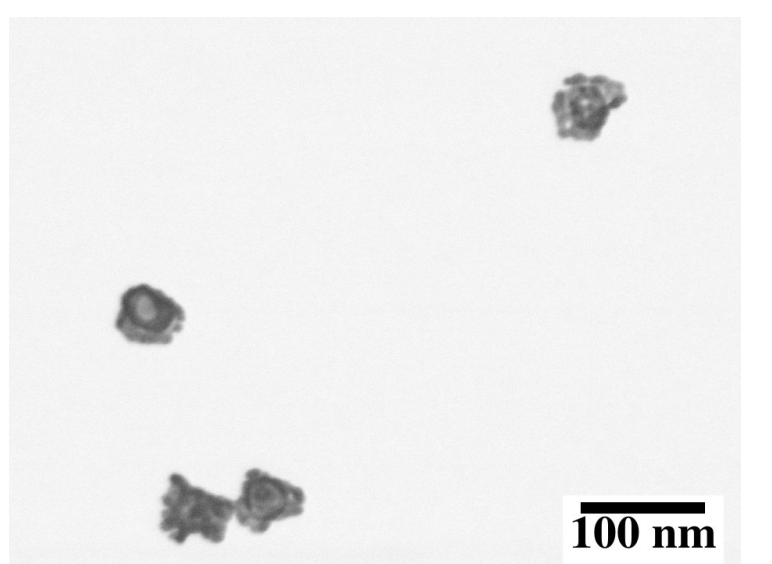

Figure 3. STEM micrograph of HGS.

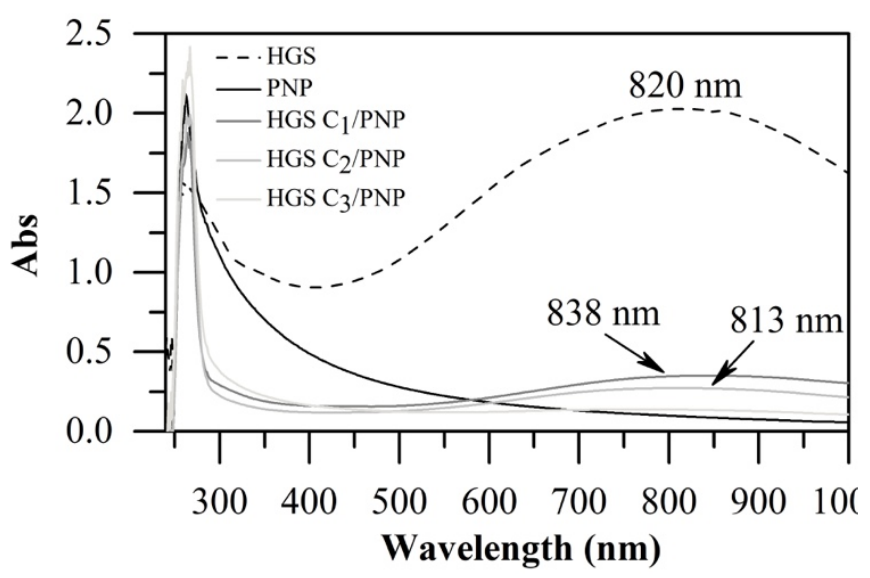

Figure 2. UV-VIS Absorption spectra for HGS, PNP, and HGS-PNP.

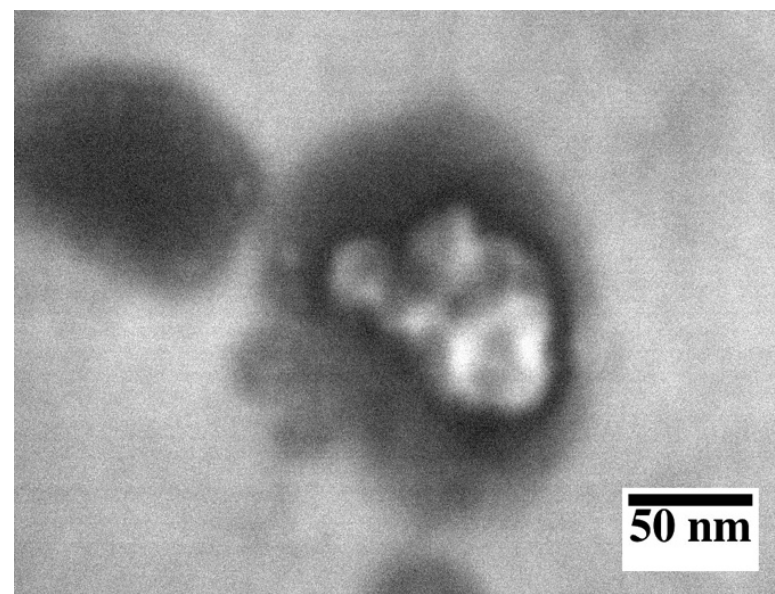

Figure 4. STEM micrograph of HGS-PNP. 